\title{
Establishment and Characterization of a Lactating Caprine Mammary Gland Luminal Epithelial Cell Line
}

\author{
Mahipal Singh ${ }^{1}$, Benjamin Hortman ${ }^{1}$, Venkata Degala ${ }^{1}, \&$ Xiaoling $\mathrm{Ma}^{1}$ \\ ${ }^{1}$ Animal Science Division, Agricultural Research Station, Fort Valley State University, Fort Valley, USA \\ Correspondence: Mahipal Singh, Animal Science Division, Agricultural Research Station, 1005 State University \\ Drive, Fort Valley State University, Fort Valley, GA 31030, USA. E-mail: singhm@fvsu.edu
}

Received: June 21, 2019

doi:10.5539/ijb.v11n4p36
Accepted: July 10, 2019

Online Published: July 16, 2019

\begin{abstract}
Mammary gland is a defining characteristic of mammalian species which produces nutritious milk and plays a major role in the development of newborns. The gland contains a series of ducts and crevices leading back to alveoli, which contain milk producing cells called luminal epithelial cells. These cells, if cultured in-vitro, can be utilized to explore the metabolic processes occurring during milk production. The knowledge thus gained can be used to manipulate the system to enhance milk production and/or modify its composition. The main objective of this study was to establish a luminal epithelial cell-line from a lactating goat. Explant culture technique was used to produce primary cells from the mammary tissue of a 4-year-old lactating Saanen goat. The outgrowing cells were purified by selective trypsinization to remove fibroblast cells in 3-4 serial passages. The purified cell cultures exhibited cobblestone morphology, typical of the mammary epithelial cells, formed clear islands when plated in low density, and exhibited dome-shaped structures, if cultured for extended time. The cells stained positive with anti-human cytokeratin 18 antibodies, confirming their epithelial nature. Cell cultures also stained positive with rabbit anti-bovine $\beta$-lactoglobulin antibodies, indicating milk production in these cells. The cell-line has potential as an in-vitro cell model to understand signaling during milk synthesis, mammary gland development, and testing DNA constructs for therapeutic protein secretion in milk, prior to production of transgenic goats.
\end{abstract}

Keywords: Goat, Epithelial Cells, In-Vitro Culture, Cytokeratin 18, Mammary Gland, Immunofluorescence, Milk Protein, $\beta$-Lactoglobulin

\section{Introduction}

Development of mammary glands, for suckling of their young infants, is an important characteristic of all mammals. The gland is a modified cutaneous exocrine organ which has various physiological, immunological and biochemical functions. It is like a double-tube and is specialized in producing milk which contains the essential nutrients to support neonates and young infants for their nutritional and developmental needs. Its development is very well coordinated and undergoes changes facilitating glandular development, differentiation, expression of secretary products, and finally down regulation and cessation of lactation and glandular remodeling. The mammary gland is comprised of a series of ducts and crevices, leading back to alveoli, which contain the milk producing cells known as luminal epithelial cells. Although the mammary gland evolved to facilitate neonate and young infant nourishment, humans have practiced it for bulk production of milk for their own consumption in some livestock species. Developments in biotechnology research further made it possible to use mammary glands as bioreactors, for secretion of therapeutic proteins in milk, for use in human and veterinary medicine (Bosze, Baranyi, \& Whitelaw, 2008; Carneiro et al., 2018; Ziomek, 1998). These applications of therapeutic protein production in milk require manipulation of genomes and characterization of modified genes, in in-vitro culture systems, prior to the production of embryos and live offspring.

The cells of mammary glands are very sensitive to toxic agents, leading to breast cancers in human beings (Fenton, 2006; Osborne, Rudel, \& Schwarzman, 2015; Paulose, Speroni, Sonnenschein, \& Soto, 2015). Thus, the study of development, differentiation, and regulation of mammary gland has gained significant attention. The progress in in-vitro culture systems, in last few decades, has made it possible to establish mammary epithelial cell lines (MECs) which could be used to study various aspects of mammary gland development, lactation physiology, and gene expression (Zhou, Chen, Zou, Zheng, \& Chen, 2019). MECs have been established in-vitro in several species including goat (Tong, Li, Gao, \& Yin, 2012), sheep (Ilan, Barash, Gootwine, \& Shani, 1998), yak (Fu, Chen, 
Xiong, Lan, \& Li, 2014), buffalo (Anand et al., 2012; Kaushik et al., 2013), and bovine (Hu et al., 2009). However, none of these cell-lines is commercially available, to our knowledge. Even, stable cell-lines have been developed in some cases (Garbe et al., 2014; Joshi, Modur, Cheng, Robinson, \& Rao, 2017; Shi et al., 2014). However, all these cell-lines have been shown to lose their milk producing capacity after some passages, and thus do not reflect the in-vivo physiology of mammary cells. Therefore, the primary cells at early passages are obvious choices, to study mammary gland function. The goal of this study was to establish and characterize a mammary epithelial cell line from American Saanen goats, which can be used for lactation, mastitis, as well as for testing DNA constructs for milk-specific protein secretion to produce transgenic goats in future studies.

\section{Material and Methods}

\subsection{Collection of Mammary Tissue}

Mammary gland tissue was collected from the meat processing facility (slaughter house) of the university from a 4-year old lactating American Saanen goat, around 80 days of lactation. Since the tissues were collected from slaughterhouse and animals were not specially sacrificed for this work, ethical approval was not needed. The tissue was brought to the laboratory on ice, cleaned with copious amount of water to remove traces of the milk, and processed within $2 \mathrm{~h}$ of collection.

\subsection{Culturing Primary Cells}

Goat mammary epithelial cells (gMECs) were cultured using an explant culture method as described previously for skin fibroblast cells (Singh \& Sharma, 2011), except that mammary epithelial cell basal medium (cat. 814-500) supplemented with growth supplements (cat. 815-GS) was used (Cell Applications Inc., San Diego, CA). In brief, small $3-5 \mathrm{~mm}^{2}$ explants were excised from the mammary gland tissue and adhered onto $90 \mathrm{~mm}$ dishes. Growth media was then slowly added to the dishes and the explants cultured at $37^{\circ} \mathrm{C}$ in a humidified $5 \% \mathrm{CO}_{2}$ incubator. Media was changed every 3-4 days. The dishes were observed every alternate day after 4 days of culturing and any explant found dislodged was removed. Once the outgrowth around explants reached $80-90 \%$ confluence, the cells were trypsinized and plated on fresh dishes.

\subsection{Purification of gMECs}

Once the passage one (p1) cell monolayer cultures reached about $80-90 \%$ confluence, they were purified by progressive removal of fibroblast cells by partial treatment with $0.125 \%$ trypsin solution. In brief, the cell monolayers were washed twice with phosphate buffered saline (PBS), treated with trypsin solution, incubated in $\mathrm{CO}_{2}$ incubator at $37^{\circ} \mathrm{C}$ (or at room temperature) and watched for detachment of fibroblast cells (which detach first, while epithelial cells still remain attached). The detached cells along with trysin solution were carefully pipetted out. The dishes were washed quickly once with PBS solution and trypsinized again. The cells were collected by centrifugation at $1000 \mathrm{rpm}$ for $7 \mathrm{~min}$, and the pellet dissolved in complete growth medium and incubated again in $\mathrm{CO}_{2}$ incubator at $37^{\circ} \mathrm{C}$. When the cultures reached about $80-90 \%$ confluence, the process was repeated until pure epithelial cell cultures were obtained. The pure cell cultures were frozen in liquid nitrogen at $1 \times 10^{5} \mathrm{cell} / \mathrm{s} / \mathrm{mL}$ in several vials until used for further analysis.

\subsection{Immunofluorescence}

gMECs and the fibroblast cells (as control) were cultured in $800 \mu \mathrm{L}$ of media in 4-well chamber glass slides (BD Biosciences, Bedford, MA) in a $5 \% \mathrm{CO}_{2}$ incubator. A concentration of 100,000 cells $/ \mathrm{mL}$ was used for each well. For immunofluorescence, Image It Fix Perm kit from Molecular Probes was used for labeling and detection (Life Technologies Corp. Carlsbad, CA). After overnight culture, the media was removed and the cells were fixed by incubating at room temperature for $15 \mathrm{~min}$ with $200 \mu \mathrm{L}$ of fixative solution in each well. The cells were washed thrice with $400 \mu \mathrm{L}$ of wash buffer for $5 \mathrm{~min}$ each and then incubated with $200 \mu \mathrm{L}$ of permeabilization solution for $15 \mathrm{~min}$. The cells were washed again thrice and were incubated with $200 \mu \mathrm{L}$ of blocking solution for $1 \mathrm{~h}$. After removing the blocking solution, $200 \mu \mathrm{L}$ of 1:100 dilution of anti-human cytokeratin 18 AF488 antibodies (cat. 539815-80; Life Technologies, Corp. Carlsbad, CA) for testing epithelial nature of cells or 1:25 dilution of rabbit anti-bovine beta-lactoglobulin polyclonal antibodies labeled with FITC (cat. LS-c210696; LifeSpan Biosciences Inc., Seattle, WA, USA), for testing presence of $\beta$-lactoglobulin (a milk protein), were added and the cells incubated at room temperature for $2 \mathrm{~h}$ in dark (or overnight at $4{ }^{\circ} \mathrm{C}$ ). The antibody dilutions were made in blocking solution. All cells were also stained with DAPI for nuclear staining. After staining the cells were washed thrice. The excess moisture was removed by tilting the chamber slide to side and finally the chamber was carefully removed from the glass slide. On the slide, a drop of ProLong antifade mount was added in the middle of each chamber and covered with a coverslip. The slide was cured overnight by incubating in dark. The coverslip edges were sealed with nail polish, to prevent drying and movement under microscope. The images were captured after 
$24 \mathrm{~h}$ using fluorescence microscope. All incubation steps after culturing were done at room temperature unless otherwise indicated.

\section{Results}

\subsection{Morphology and Growth Characteristics of gMEC Line}

To obtain epithelial cell cultures, we used a 4-year old lactating Saanen goat, which contained active milk production capacity and had very well developed canal system in the mammary tissue, as shown (Figure 1, panel B). Using the explant culture method, we were able to see outgrowth around explants within 1-2 weeks of culture (Figure 1, panel C). Out of 87 explants cultured, 43 exhibited clear outgrowth. Both fibroblast and epithelial cells were observed in cultures (Figure 1, panels D \& E). Using selective trypsinization of the cell cultures, we were able to obtain nearly pure mammary epithelial cell cultures after 3-4 passages. It is known that fibroblast cells detach from the plastic surfaces slightly earlier than epithelial cells, upon trypsinization. This characteristic of the differences in rates of cell detachment from plastic surfaces was used to obtain fibroblast free cultures of goat epithelial cells. Mammary epithelial cells growing as a monolayer on plastic surface were then observed for their morphological characters. The cell cultures show a cobble-stone morphology, typical of mammalian epithelial cells (Figure 1, panel F). When the cells were plated in small densities, they formed clear islands (Figure 1, panel $\mathrm{H} \& \mathrm{I}$ ). If the cells are grown for extended period of time, they exhibited dome-shaped structures (Figure 1, panel G). Cell cultures when grown in epithelial culture media, with growth supplements, exhibited tiny milk and lipid droplets (Figure 3). Duct-like structures were also clearly visible in the monolayers in high magnification (Figure $3)$.
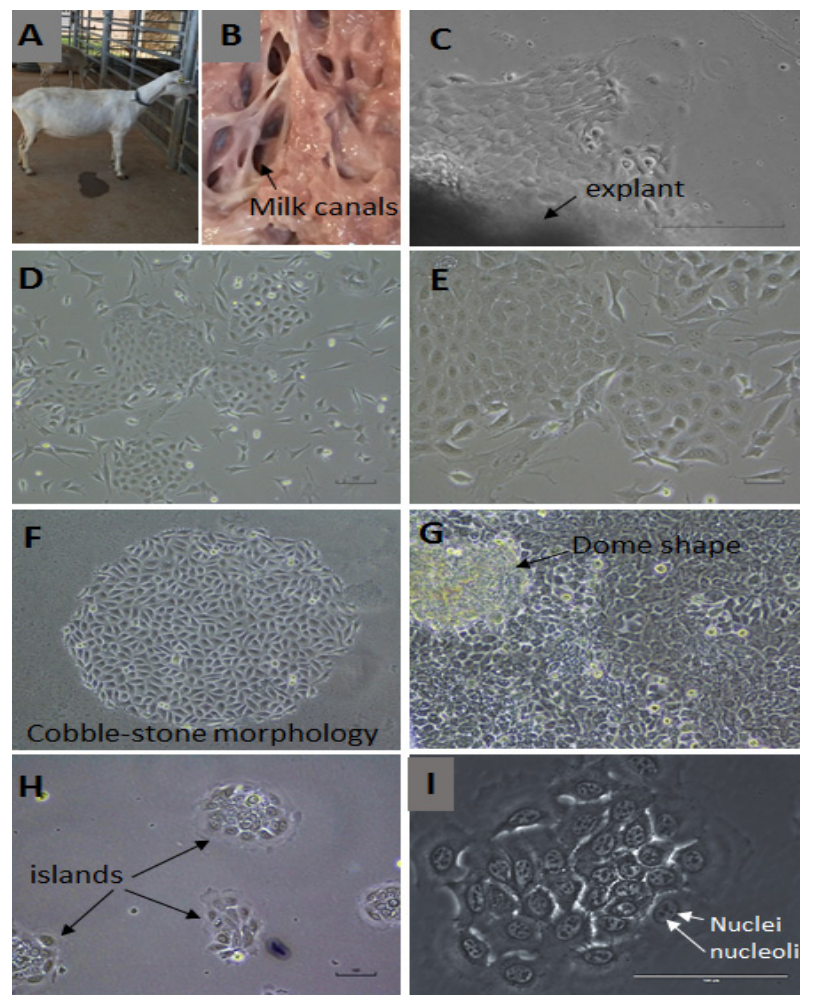

Figure 1. Morphological observations of gMECs growing as a monolayer on plastic surface

(A, a lactating dairy goat; $\mathbf{B}$, a section of the inner mammary gland like a honey comb structure exhibiting milk canals (whitish part in canals is leftover milk); Phase contrast images of, $\mathbf{C}$, primary outgrowth around mammary tissue on day11 of in-vitro culture, x200 magnification; D, mixed colonies of epithelial and fibroblast cells in passage one (p1) cultures on day 4 of cell culture (note the cobble-stone morphology of epithelial cells but elongated fibrous morphology of fibroblasts), x100; E, same as "D" but x200 magnification exhibiting clear islands and visible nuclei and nucleoli within them; F, a single colony of gMECs exhibiting cobble-stone morphology, x100; G, a dome shaped structure in gMEC cultures, x200 observed only in post-confluent cultures at around day $7 ; \mathbf{H}$, gMEC islands clearly visible when cells are plated at low density (p1, day 3 cultures), x100; I, same as " $\mathrm{H}$ " but $\mathrm{x} 400$ magnification with clear round nuclei and visible nucleoli.) 


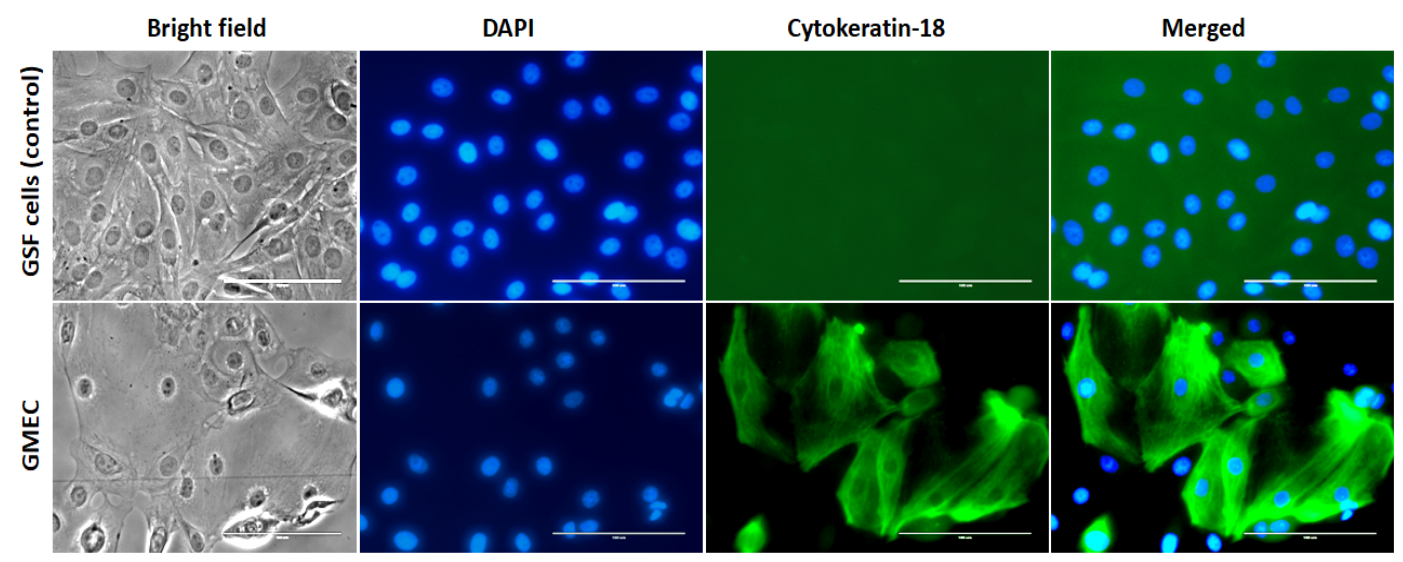

Figure 2. Immunofluorescence staining of cells with epithelial cell-specific marker antibodies

(Upper panels represent goat skin fibroblast (GSF) cells as control; whereas lower panel represent gMECs. The cells were separately stained with cytokeratin 18 antibodies labelled with FITC, for epithelial cell staining, and DAPI for nuclear staining. Images captured in Evos Fluorescence phase contrast inverted microscope, $\mathrm{x} 400$ magnifications. Right 3 panels show images under UV light with DAPI or GFP filter, whereas left most panels are their respective bright field images. Blue, DAPI for nuclear stain; Green, cytokeratin 18-FITC for epithelial cell staining. Images captured after $24 \mathrm{~h}$ of staining. Antibody dilution used was 1:100.)

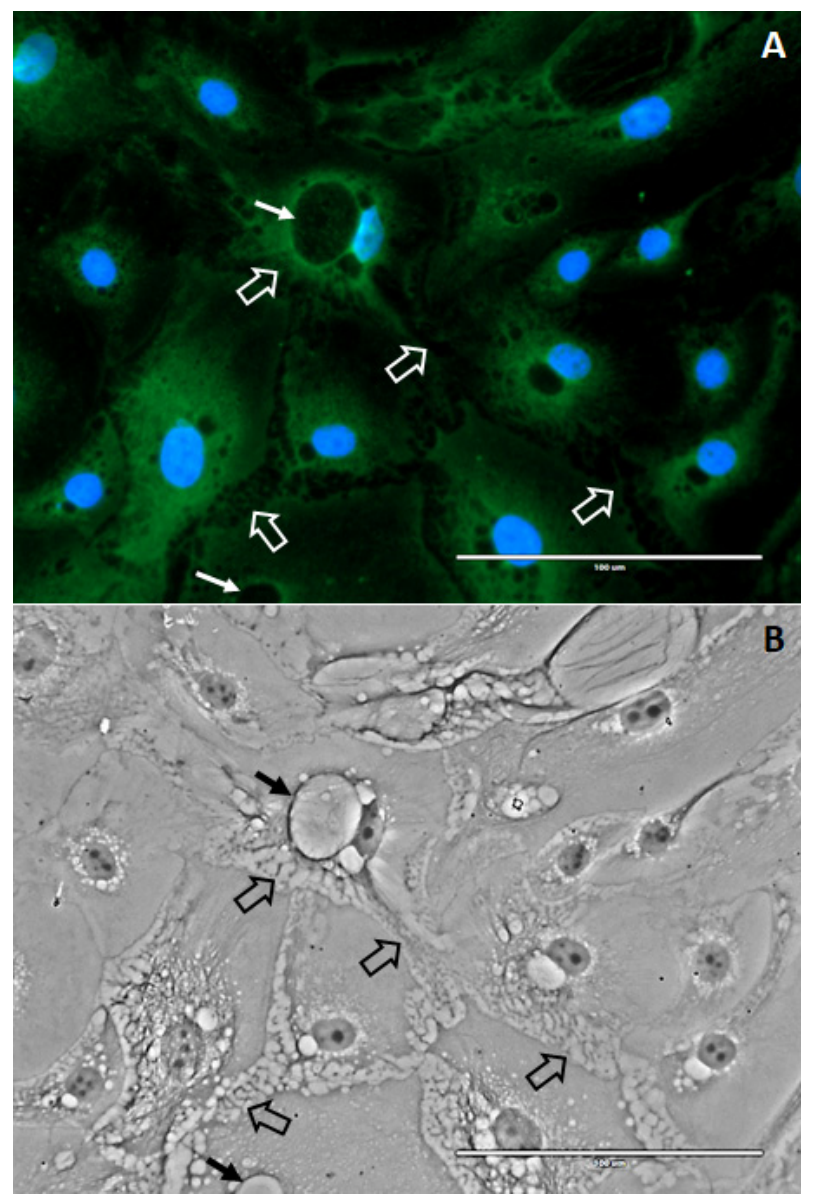

Figure 3. Secretion of a milk protein $\beta$-lactoglobulin in gMECs

(A, Immunofluorescence of gMECs using rabbit anti-bovine $\beta$-lactoglobulin antibodies and DAPI nuclear stain;

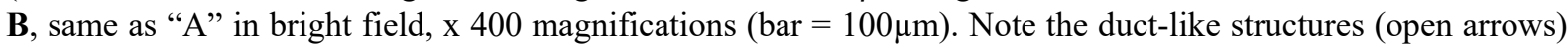
showing small milk-lipid droplets along the epithelial cell borders. Closed arrow show large lipid drop like structures.) 


\subsection{Cytokeratin 18 Expression in gMECs}

The gMEC line established in this study, when analyzed for presence or absence of cytokeratin 18, a defined marker of epithelial cells (which is absent in fibroblast cells), exhibited clear positive signal with anti-human cytokeratin 18 antibodies (as shown in Figure 2), confirming their epithelial nature. No positive fluorescence signal was detected in similarly stained fibroblast cells, which were used as controls (Figure 2, upper panel). We observed that some cells in gMEC cultures did not stain or stained poorly. It is possible that they had very low levels of expression of cytokeratin 18. It is also possible that since these cells were stained with anti-human antibodies due to non-availability of anti-goat specific antibodies, low levels of marker expression remain undetectable. All cells stained clearly with DAPI exhibiting clear nucleus (Figure 2).

\section{3 -Lactoglobulin Expression in gMEC Line}

When the cell monolayers were analyzed for presence of $\beta$-lactoglobulin (a unique milk protein) by immunofluorescence, using rabbit anti-bovine $\beta$-lactoglobulin polyclonal antibodies, we were able to show that cells stained positive, indicating the presence of $\beta$-lactoglobulin protein expression in the monolayer cells (Figure 3 , panel A). High magnification images in phase contrast bright field microscopy clearly show duct-like structures (Figure 3, panel B) which seems to carry mammary epithelial cell secreted tiny milk-lipid droplets.

\section{Discussion}

Development of mammary gland is a unique feature of mammals, which is evolved to synthesize and secrete milk, to nourish the new born. In the initial stages of life, mother's milk is a complete food, providing not only the required nutrition but also immunity. Development of glandular tissue, milk synthesis in epithelial cells of the alveoli, and its secretion is very well coordinated by various signaling molecules. Studying various functional aspects of these signaling events is difficult and time consuming in live animals. Scientists have developed in-vitro culture systems and established mammary epithelial cell-lines in various animal systems (Tong et al., 2012). In this study we established mammary epithelial cell-line from American Saanen goats using explant culture method. We purified the milk producing epithelial cells, from mixed primary cultures, by serial-passaging using a selective trypsin treatment, to remove the fibroblast cells, prior to collecting pure epithelial cells. The cells exhibit cobblestone morphology and express cytokeratin 18 protein, confirming their epithelial nature. These cells also produce $\beta$-lactoglobulin, a milk protein, as demonstrated by their positive signal with anti- $\beta$-lectoglobulin antibodies. This cell-line will be used as an in-vitro cell model to understand signaling during milk synthesis and testing DNA constructs for therapeutic protein secretion in milk, prior to nuclear transfer of modified genomes, to produce transgenic goats.

\section{Conclusion}

In conclusion, we have established a goat mammary epithelial cell-line from saanen goats by using an explant culture system and selective trypsinization of the cultures to remove contaminating fibroblasts.

\section{Acknowledgements}

This work was partly supported by the USDA National Institute of Food and Agriculture, Evans-Allen project number 1009701 to MS. Part of this work was presented in the annual meeting of the Society for In Vitro Biology, Tampa, FL, June 8-12, 2019.

\section{Conflict of interests}

The authors declare that there is no conflict of interests regarding the publication of this paper.

\section{References}

Anand et al. (2012). Establishment and characterization of a buffalo (bubalus bubalis) mammary epithelial cell line. PloS One, 7(7), e40469.

Bosze, Z., Baranyi, M., \& Whitelaw, C. B. (2008). Producing recombinant human milk proteins in the milk of livestock species. Advances in Experimental Medicine and Biology, 606, 357-393.

Carneiro et al. (2018). Milk from transgenic goat expressing human lysozyme for recovery and treatment of gastrointestinal pathogens. European Journal of Pharmaceutical Sciences: Official Journal of the European Federation for Pharmaceutical Sciences, 112, 79-86.

Fenton, S. E. (2006). Endocrine-disrupting compounds and mammary gland development: Early exposure and later life consequences. Endocrinology, 147(6 Suppl), S18-24.

Fu, M., Chen, Y., Xiong, X., Lan, D., \& Li, J. (2014). Establishment of mammary gland model in vitro: Culture and evaluation of a yak mammary epithelial cell line. PloS One, 9(12), e113669. 
Garbe et al. (2014). Immortalization of normal human mammary epithelial cells in two steps by direct targeting of senescence barriers does not require gross genomic alterations. Cell Cycle (Georgetown, Tex.), 13(21), 34233435.

Hu et al. (2009). In vitro culture and characterization of a mammary epithelial cell line from chinese holstein dairy cow. PloS One, 4(11), e7636.

Ilan, N., Barash, I., Gootwine, E., \& Shani, M. (1998). Establishment and initial characterization of the ovine mammary epithelial cell line NISH. In Vitro Cellular \& Developmental Biology. Animal, 34(4), $326-332$.

Joshi, P. S., Modur, V., Cheng, J., Robinson, K., \& Rao, K. (2017). Characterization of immortalized human mammary epithelial cell line HMEC 2.6. Tumour Biology: The Journal of the International Society for Oncodevelopmental Biology and Medicine, 39(10), 1010428317724283.

Kaushik et al. (2013). Isolation, characterization, and EGFP expression in the buffalo (bubalus bubalis) mammary gland epithelial cell line. In Vitro Cellular \& Developmental Biology. Animal, 49(1), 1-7.

Osborne, G., Rudel, R., \& Schwarzman, M. (2015). Evaluating chemical effects on mammary gland development: A critical need in disease prevention. Reproductive Toxicology (Elmsford, N.Y.), 54, 148-155.

Paulose, T., Speroni, L., Sonnenschein, C., \& Soto, A. M. (2015). Estrogens in the wrong place at the wrong time: Fetal BPA exposure and mammary cancer. Reproductive Toxicology (Elmsford, N.Y.), 54, 58-65.

Shi et al. (2014). Establishment and characterization of a dairy goat mammary epithelial cell line with human telomerase (hT-MECs). Animal Science Journal = Nihon Chikusan Gakkaiho, 85(7), 735-743.

Singh, M., \& Sharma, A. K. (2011). Outgrowth of fibroblast cells from goat skin explants in three different culture media and the establishment of cell lines. In Vitro Cellular \& Developmental Biology. Animal, 47(2), 83-88.

Tong, H. L., Li, Q. Z., Gao, X. J., \& Yin, D. Y. (2012). Establishment and characterization of a lactating dairy goat mammary gland epithelial cell line. In Vitro Cellular \& Developmental Biology. Animal, 48(3), 149155 .

Zhou, J., Chen, Q., Zou, Y., Zheng, S., \& Chen, Y. (2019). Stem cells and cellular origins of mammary gland: Updates in rationale, controversies, and cancer relevance. Stem Cells International, 4247168.

Ziomek, C. A. (1998). Commercialization of proteins produced in the mammary gland. Theriogenology, 49(1), 139-144.

\section{Copyrights}

Copyright for this article is retained by the author(s), with first publication rights granted to the journal.

This is an open-access article distributed under the terms and conditions of the Creative Commons Attribution license (http://creativecommons.org/licenses/by/4.0/). 